\title{
Convolution approximation and shift approximation
}

\author{
by
}

J. MIKUSIŃSKI (Katowice)

Part I. Convolution approximation

1. In this paper, the convolution

$$
\int_{0}^{t} g(t-\tau) k(\tau) d \tau
$$

will be denoted by $g k$ (instead of the usual notation $g * k$ ). The ordinary product of two functions will be denoted, on writing explicitely the arguments, e. g. $g(t) k(t)$. Such a notation is also used in my book [2].

Let $C[0, T](0<T<\infty)$ be the class of real continuous functions on the interval $[0, T], C_{0}[0, T]$ the subclass of $C[0, T]$ of functions which vanish at 0 , and $O_{0}^{\infty}[0, T]$ the class of infinitely derivable functions in $[0, T]$ which vanish at 0 together with all their derivatives.

THEOREM I. For any fixed $g \in C[0, T]$ which does not vanish identically in the right neighbourhood of 0 , the set of convolutions $g k$ with $k \in C_{0}^{\infty}[0, T]$ is dense in $C_{0}[0, T]$.

This theorem is, in fact, due to Foiaş [1], who needed it to prove that the set of continuous functions is dense in the space of operators. However, Foiaş formulated it in a slightly different form: For any fixed $g \in L^{1}[0, T]$, not vanishing almost everywhere at the neighbourhood of 0 , the set of convolutions $g k$ with $k \in O[0, T]$ is dense in $L^{1}[0, T]$. Another formulation of Theorem $I$ is given in the paper [3]. There it is proved that, for any fixed $g \in C_{0}[0, T]$, non-vanishing in the neighbourhood of 0 , the set of convolutions $g k$ with absolutely continuous functions $k$ is dense in $C[0, T]$. The proof given in [3] can be used for Theorem $\mathrm{I}$; the only needed modifications are the following: one assumes that $g \in C[0, T]$ (instead of $g \in C_{0}[0, T]$ ), $k_{n}, k \in C_{0}^{\infty}[0, T]$ (instead of $k_{n}, k \in A C$ (absolutely continuous functions)) and one has $k^{\prime} \in O_{0}^{\infty}[0, T]$ (instead of $k^{\prime} \in L^{1}[0, T]$ ).

Evidently, Theorem $I$ is a little stronger than my earlier formulation in [3]. It is easy to see that it is also stronger than the formulation 
in Foiaş's paper [1]. In fact, let $g \in L^{1}[0, T]$ and let

$$
h=\int_{0}^{t} g(\tau) d \tau .
$$

By Theorem I, the set of convolutions $h k^{\prime}$, where $k \epsilon O_{0}^{\infty}[0, T]$ is dense in $C_{0}[0, T]$. Since $h k^{\prime}=g k$, we may also say that the set of convolutions $g k$ where $k \epsilon C_{0}^{\infty}[0, T]$ is dense in $C_{0}[0, T]$. A fortiori, the set of convolutions $g k$ where $k \in C[0, T]$ is dense in $C_{0}[0, T]$. Since $C_{0}[0, T]$ is dense in $L^{1}[0, T]$, this set is dense in $L^{1}[0, T]$.

In the Part $I$ of this paper we are going to strengthen Theorem I in three steps. In this way we shall obtain Theorems II, III and IV, each of them being stronger than the preceding one.

THEOREM II. Given any fixed $g \in O[0, T]$ which does not vanish identically in the right neighbourhood of 0 , the set of convolutions gle with $k_{\epsilon} O_{0}^{\infty}[0, T]$ is dense in $C_{0}^{\infty}[0, T]$.

Proof. Let $f \epsilon C_{0}^{\infty}[0, T]$ and let $\varepsilon_{n}$ be a sequence of positive numbers, tending to 0 as $n \rightarrow \infty$. By Theorem I, there exists, for every positive integer $n$, a function $k_{n} \in C_{0}^{\infty}[0, T]$ such that

This implies that

$$
\left|g k_{n}^{(n)}-f^{(n)}\right|<T^{-n} \varepsilon_{n}
$$

$$
\left|g k_{n}^{(i)}-f^{(i)}\right|<T^{-i} \varepsilon_{n} \quad \text { for } \quad i=0,1, \ldots, n .
$$

Thus, for any fixed $i$, the sequence $g k_{n}^{(i)}$ converges uniformly to $f^{(i)}$, as $n \rightarrow \infty$. This means that $g k_{n} \rightarrow f$ in the topology of $C_{0}^{\infty}[0, T]$, which proves Theorem II.

The fact that Theorem II is stronger than Theorem I follows from the remark that $C_{0}^{\infty}[0, T]$ is dense in $C_{0}[0, T]$.

Let $C[0, \infty)$ denote the class of continuous functions in the interval $[0, \infty)$ and $C_{0}^{\infty}[0, \infty)$ the class of indefinitely derivable functions in that interval. We say that a sequence of functions from the class $O[0, \infty)$ or from the class $C_{0}^{\infty}[0, \infty)$ is convergent in $O[0, \infty)$ or in $O_{0}^{\infty}[0, \infty)$ respectively, if the corresponding sequence of functions restricted to any bounded interval $[0, T]$ is convergent in the proper topology, of $C[0, I]$ or $O_{0}^{\infty}[0, T]$.

THEOREM III. For any fixed $g \in C[0, \infty)$ which does not vanish identically in the right neighbourhood of 0 , the set of convolutions gk with $k \epsilon C_{0}^{\infty}[0, \infty)$ is dense in $O_{0}^{\infty}[0, \infty)$.

Proof. Let $f_{\epsilon} O_{0}^{\infty}[0, \infty)$. By Theorem II, there is, for any positiv integer $p$, a sequence of functions $k_{p n} \epsilon C_{0}^{\infty}[0, \infty)$ such that $\left|g k_{p m}^{(i)}-f^{(i)}\right|<\varepsilon_{i}$ in $[0, p]$, where $0<\varepsilon_{i n} \rightarrow 0$ as $n \rightarrow \infty$. This implies that the diagonal sequence $g k_{n n}^{(i)}$ converges to $f^{(i)}$ uniformly in every interval $[0, T]$ $(0<T<\infty)$, which proves Theorem III.

Evidently, Theorem III reduces to Theorem II, when restricting the considered functions from $[0, \infty)$ to $[0, T]$.

2. Let $M_{+}$denote the space of all operators $a=p / q$ (see [2]), where $p, q \in C[0, \infty)$ and $q$ does not vanish identically in any right neighbourhood of 0 . We say that a sequence $a_{n} \in M_{+}$converges in $M_{+}$, if there is a function $q \in C[0, \infty)$ non-vanishing identically in any neighbourhood of 0 , such that all the operational products $a_{n} q$ are functions of class $C[0, \infty)$ and $a_{n} q$ converges almost uniformly in $C[0, \infty$ ) (i. e., uniformly in every bounded interval $[0, T])$. We say that an operator $a \in M_{+}$does not vanish in the right neighbourhood of 0 , if it is of the form $p / q$, where both $p$ and $q$ are functions which do not vanish identically in the right neighbourhood of 0 (see [4]).

THEOREM IV. For any fixed operator $g \in M_{+}$which does not vanish in the right neighbourhood of 0 , the set of elements $g k$, where $k \epsilon C_{0}^{\infty}[0, \infty)$, is dense in $C_{0}^{\infty}[0, \infty)$.

Proof. Let $f \in C_{0}^{\infty}[0, \infty)$. There is a function $q \in C[0, \infty)$, non-vanishing identically in the right neighbourhood of 0 , such that $g q \in C[0, \infty)$; evidently the function $g q$ does not vanish either identically in the right neighbourhood of 0 . Thus, by Theorem III, there are functions $k_{n} \in C_{0}^{\infty}[0, \infty)$ such that the sequence $(g q) k_{n}$, i. e., $g\left(q k_{n}\right)$, converges to $f$ in the topology of $C_{0}^{\infty}[0, \infty)$. Since $q k_{n} \in C_{0}^{\infty}[0, \infty)$, Theorem IV is proved.

In order to see that Theorem IV is stronger than Theorem III, it suffices to observe that $C[0, \infty)$ is a subset of $M_{+}$.

\section{Part II. Shift approximation}

3. Let $S$ be a linear subspace of $M_{+}^{*}$ containing $C_{0}^{\infty}[0, \infty)$, with the following properties:

$1^{\circ} S$ is a locally convex topological space such that every sequence $f_{n} \epsilon C_{0}^{\infty}[0, \infty)$ which converges in $C_{0}^{\infty}[0, \infty)$ converges also in $S$ to the same limit; moreover, every sequence $f_{n} \in S$ which converges in $S$, converges also in $M_{+}$to the same limit; finally, we assume that $O_{0}^{\infty}[0, \infty)$ is dense in $S$;

$2^{\circ}$ If $f \in S$, then $h^{\lambda} f \in S$ ( $h$ shift-operator) for every $\lambda \geqslant 0$. In the topology of $S, h^{\lambda} f$ is a continuous function of $\lambda$ in the interval $0 \leqslant \lambda<\infty$;

$3^{\circ}$ There is a family of semi-norms $\|f\|_{\alpha}$ with $\alpha \in A$ such that: for any $\alpha \in A$ there is a number $\lambda_{\alpha}>0$ such that $\lambda>\lambda_{a}$ implies $\left\|h^{\lambda} f\right\|_{\alpha}=0$ for every $f \in S$. 
We are going to give a few examples of the space $s$.

(i) Space $O_{0}^{\infty}[0, \infty)$. Here, we have

$$
h^{\lambda} f=\left\{\begin{array}{cll}
f(t-\lambda) & \text { for } & t \geqslant \lambda, \\
0 & \text { for } & 0 \leqslant t<\lambda .
\end{array}\right.
$$

For $A$, we can take the set of pairs $\alpha=(p, r)$ of integers $p, r$ $(p \geqslant 0, r \geqslant 1)$ and then let

$$
\|f\|_{(p, r)}=\max _{0 \leqslant \leqslant_{\xi} t \leqslant r}\left|f^{(p)}(t)\right| .
$$

(ii) Space $C_{0}^{p}[0, \infty)$. The elements of this space are functions in $[0, \infty)$, derivable up to the order $p$ in that interval, and vanishing together with these derivatives at 0 . A sequence $f_{n} \epsilon C_{0}^{p}[0, \infty)$ is said to converge in $C_{0}^{p}[0, \infty)$, if for any $i=0, \ldots, p$, the sequence $f_{n}^{(i)}$ converges uniformly in every interval $[0, T](0<T<\infty)$. Formula (1) holds also in the actual case. For $A$, we can take $\alpha=(i, r)$ of integers $i, p(0 \leqslant i \leqslant p, r \geqslant 1)$ and then let

$$
\|f\|_{(i, r)}=\max _{0 \leqslant t \leqslant r} f^{(i)}(t) \mid .
$$

(iii) Space $C_{0}[0, \infty)$. Its elements are continuous functions in $[0, \infty)$, vanishing at 0 . This is a particular case of the preceding example (with $p=0$ ). Actually, for $A$ we can take the set of all positive integers $\alpha=r$ and let

$$
\|f\|_{r}=\max _{0 \leqslant t \leqslant r}|f(t)| \text {. }
$$

(iv) Space $L^{p}[0, \infty), p \geqslant 1$. The elements of this space are functions which are locally $p$-integrable in $[0, \infty)$, i. e. $p$-integrable on every bounded interval $[0, T]$. Formula (1) holds also in the present case. For $A$, we can take the set of all positive integers and let

$$
\|f\|_{r}=\sqrt{\int_{0}^{r}|f(\tau)|^{p} d \tau .}
$$

(v) Space $D_{+}^{\prime}$. As elements of this space we take the distributions whose support lies on $[0, \infty)$. It turns out to say that these elements are distributions defined on the whole line $(-\infty, \infty)$ and vanish in $(-\infty, 0)$. In particular, continuous functions in $(-\infty, \infty)$, vanishing in $(-\infty, 0)$, are distributions. In order to imbed $O_{0}^{\infty}[0, \infty)$ into $D_{+}^{\prime}$, we extend the definition of $f \in C_{0}^{\infty}[0, \infty)$ onto the negative part of the real axis, assuming that $f$ vanishes on that part. Evidently, formula (1) makes sense in the case of $D_{+}^{\prime}$. For $A$, we can take the set of all smooth (infinitely derivable) functions $\alpha$ of bounded support (vanishing outside a bounded interva1). Then we let for $f \in D_{+}^{\prime}$,

$$
\|f\|_{\alpha}=\left|\int_{-\infty}^{\infty} f(t) \alpha(t) d t\right|
$$

(vi) Space $M_{q}$. The elements of this space are operators (elements of $M_{+}$) which can be represented in the form $f=p / q$, where $p, q \in O[0, \infty)$ and $q$ does not vanish identically in the right neighbourhood of 0 . The space $M_{q}$ is thus determined by the function $q$. For $A$, we can take the set of positive integers and let

$$
\|f\|_{r}=\max _{0 \leqslant t \leqslant r}|q f|
$$

( $q$ is a continuous function).

We have evidently $C_{0}^{\infty}[0, \infty) \subset C_{0}^{p}[0, \infty) \subset C_{0}[0, \infty) \subset L^{p}[0, \infty) \subset D_{+}^{\prime}$. We also have $D_{+}^{\prime} \subset M_{q}$, provided we take for $q$ a function of class $C_{0}^{\infty}[0, \infty)$; then $D_{+}^{\prime}$ is a proper subset of $M_{q}$.

THEOREM V. For every operator $g \in S$ which does not vanish in the right neighbourhood of 0 , the set of elements of the form

$$
\lambda_{1} h^{\tau_{1}} g+\ldots+\lambda_{n} h^{\tau_{n}} g
$$

where $\lambda_{i}$ and $\tau_{i}$ are real numbers, $\tau_{i}>0$, and $h$ is the shift-operator, is dense in $S$.

Proof. Let us consider the integral

$$
\int_{0}^{\infty} h^{\tau} g k(\tau) d \tau
$$

where $k \epsilon C_{0}^{\infty}[0, \infty)$. Remark that, in the interpretations $(i)-(v)$, this integral can be written in the form

$$
\int_{0}^{t} g(t-\tau) k(\tau) d \tau,
$$

because of formula (1). Thus it equals the convolution $g k$. We shall show that it equals $g k$ also in the general case. In fact, the integral

$$
\int_{0}^{b} h^{\tau} g k(\tau) d \tau
$$

is defined for every finite $b>0$, since its integrand is continuous. The value of (3) is to be considered as the limit of (4), as $b \rightarrow \infty$. The existence of that limit follows from the inequality

$$
\left\|\int_{\tau_{1}}^{\tau_{2}} h^{\tau} g k(\tau) d \tau\right\|_{\alpha} \leqslant \int_{\tau_{1}}^{\tau_{2}}\left\|h^{\tau} g\right\|_{\alpha}|k(\tau)| d \tau \quad\left(\tau_{1}<\tau_{2}\right)
$$


and from the fact that $\left\|h^{\tau} g\right\|_{a}=0$ for $\lambda_{a}<\tau_{1} \leqslant \tau \leqslant \tau_{2}$. On the other hand, by $1^{\circ}$, the limit (3) can be also considered in the operational sense, and so we see that it equals

$$
g \int_{0}^{\infty} h^{\tau} k(\tau) d \tau=g k \in S
$$

(see e.g. formula (9.1), p. 337, of [2])

Let

$$
w_{n}\left(h^{1 / n}\right)=\lambda_{1} h^{1 / n}+\lambda_{2} h^{2 / n}+\ldots+\lambda_{n^{2}} h^{n}
$$

where

$$
\lambda_{i}=\int_{(i-1) / n}^{i / n} k(\tau) d \tau \quad\left(n=1,2, \ldots, n^{2}\right)
$$

We have

$$
\left\|g k-w_{n}\left(h^{1 / n}\right) g\right\|_{\alpha} \leqslant\left\|\int_{0}^{n} h^{\tau} g k(\tau) d \tau-w_{n}\left(h^{1 / n}\right) g\right\|_{\alpha}+\left\|\int_{n}^{\infty} h g k(\tau) d \tau\right\|_{\alpha} .
$$

For $n>\lambda_{\alpha}$ the last integral vanishes, so we can write

$$
\begin{aligned}
\left\|g k-w_{n}\left(h^{1 / n}\right) g\right\|_{\alpha} & \leqslant\left\|\sum_{i=1}^{n^{2}} \int_{(i-1) / n}^{i / n}\left(h^{\tau} g-h^{1 / n} g\right) k(\tau) d \tau\right\| \\
& \leqslant \sum_{i=1}^{n^{2}} \int_{(i-1) / n}^{i / n}\left\|h^{\tau} g-h^{1 / n} g\right\|_{a} \cdot|k(\tau)| d \tau .
\end{aligned}
$$

For $\tau \geqslant(i-1) / n>\lambda_{\alpha}$ we have $\left\|h^{x} g-h^{1 / n} g\right\|_{\alpha} \leqslant\left\|h^{\tau} g\right\|_{\alpha}+\left\|h^{1 / n} g\right\|_{\alpha}=0$, it therefore suffices to consider, in (5), only expressions

$$
\left\|h^{\tau} g-\hbar^{1 / n} g\right\|_{a}
$$

with $\tau$ and $i / n$ belonging to the bounded interval $0 \leqslant \tau \leqslant \lambda_{\alpha}+1$. Since the function $h^{\lambda} g$ is supposed continuous, expression $(6)$ becomes less than any given $\varepsilon>0$ if $0 \leqslant(i-1) / n \leqslant \tau \leqslant i / n \leqslant \lambda_{a}+1$ and $n$ is sufficiently large, say $n>n_{0}$. Thus we obtain, for $n>n_{0}$,

$$
\left\|g \tau_{i}-w_{n}\left(h^{1 / n}\right) g\right\|_{\alpha} \leqslant \varepsilon \int_{0}^{\lambda_{a}+1}|k(\tau)| d \tau
$$

This proves that $w_{n}\left(h^{1 / n}\right) g \rightarrow g k$ in the topology of $S$. We can also say that the set of elements $w\left(h^{1 / n}\right) g$, where $w$ are polynomials with real coefficients, is dense in the set of convolutions $g k$. with respect to the topology of $S$. Since the set of convolutions $g k$ is dense in $C_{0}^{\infty}[0, \infty)$, by Theorem IV, and the last set is dense in $S$, by hypothesis, the set of elements $w\left(h^{1 / n}\right) g$ is dense in $S$, which proves Theorem $V$.
4. We are now going to discuss some particular cases of Theorem V. If $S$ is one of the spaces $C_{0}^{\infty}[0, \infty), C_{0}^{p}[0, \infty)$ or $C_{0}[0, \infty)$, then the hypothesis that the operator $g$ does not vanish in the right neighbourhood of 0 means that the function $g$ (actually $g$ is a function) does not vanish identically in this neighbourhood. Theorem $V$ says that, for any fixed $g$ with that property, the set of elements

$$
\lambda_{1} g\left(t-\tau_{1}\right)+\ldots+\lambda_{n} g\left(t-\tau_{n}\right)
$$

with $\tau_{i}>0$ is dense in the considered space.

This implies, in particular, that every function $f \in C[0, \infty)$ (not necessarily vanishing at 0 ) can be approximated almost uniformly in $[0, \infty)$ by sums (7) with $g_{\epsilon} C_{0}[0, \infty)$, where at most the number $\tau_{1}$ is negative, all others $\tau_{i}(i>1)$ being positive.

In fact, there is a point $t_{1}>0$ such that $g\left(t_{1}\right) \neq 0$. The function

$$
f(t)-\frac{f\left(t_{1}\right)}{g\left(t_{1}\right)} g\left(t+t_{1}\right)
$$

belongs evidently to $C_{0}[0, \infty)$ and can be therefore approximated almost uniformly by sums

$$
\lambda_{2} g\left(t-\tau_{2}\right)+\ldots+\lambda_{n} g\left(t-\tau_{n}\right) .
$$

Hence, our assertion follows, on taking $\lambda_{1}=f\left(t_{1}\right) / g\left(t_{1}\right)$ and $\tau_{1}=-t_{1}$. If we restrict the functions to a bounded interval $[0, T]$, then we obtain a theorem proved in [3].

In a similar way we can show that every function $f \in C^{p}[0, \infty)$ can be approximated almost uniformly together with their derivatives up to the order $p$ by sums (7), where at most $p+1$ numbers $\tau_{1}, \ldots, \tau_{p+1}$ are negative.

If $S=L^{p}[0, \infty)(p \geqslant 1)$, then the assumption that $g$ does not vanish in the right neighbourhood of 0 means that there is no right neighbourhood of 0 in which the function $g$ vanishes almost everywhere. Theorem V says that the set of elements $(7)$ is dense in $L^{p}[0, \infty)$. If we restrict the functions to a bounded interval $[0, T]$, then we obtain a theorem proved by Skórnik in [6].

If $S=D_{+}^{\prime}$, then Theorem $\mathrm{V}$ says that every distribution from $D_{+}^{\prime}$ can be approximated distributionally by sums (7) with any other distribution $g$ from $D_{+}^{\prime}$ which does not vanish in the right neighbourhood of 0 , and positive numbers $\tau_{i}$. In particular, it can be approximated by sums with the delta distribution: $\lambda_{1} \delta\left(t-\tau_{1}\right)+\ldots+\lambda_{n} \delta\left(t-\tau_{n}\right)$. It might to seem, at first, more paradoxal that the delta distribution $\delta(t)$ can be approximated by sums $(7)$ with positive $\tau_{i}$ and with an arbitrary given function $g \epsilon C_{0}^{\infty}[0, \infty)$.

Finally, it follows from Theorem $\mathrm{V}$ that every operator $f \in M_{+}$can be approximated operationally by sums (2) with any other operator $g \in M$ which does not vanish in the right neighbourhood of 0 . In fact, there exists 
a function $q \in C[0, \infty)$ which does not vanish identically in the right neighbourhood of 0 such that $f=p_{1} / q$ and $g=p_{1} / q$, where $p_{1} p_{2} \epsilon O[0, \infty)$. Let $M_{q}$ be the set of all operators which can be represented in the form $p / q(p \in C[0, \infty))$. By Theorem $V, f$ can be approximated by sums $(2)$ in the topology of $M_{q}$. But every sequence which converges in the topology of $M_{q}$ also converges operationally, which proves our assertion. Taking in particular $g=1$, we see that every operator from $M_{+}$can be approximated by polynomials $\lambda_{1} h^{\tau_{1}}+\ldots+\lambda_{n} h^{\tau_{n}}$ of the shift-operator with positive $\tau_{i}$.

5. We have considered, so far, functions, distributions and operators defined on the one-dimensional real space $R$. However, all the theorems can also be interpreted in the Euclidean space $R^{m}$ of any number of dimensions. Then by an interval $[0, T]$ we understand the set of points $t=\left(t_{1}, \ldots, t_{m}\right)$ whose coordinates satisfy the inequalities $0 \leqslant t_{i} \leqslant T_{i}$, where $T=\left(T_{1}, \ldots, T_{m}\right)$. Similarly, the interval $[0, \infty)$ means the set of points $t$ with $t_{i} \geqslant 0$. By the convolution

$$
\int_{0}^{t} g(t-\tau) k(\tau) d \tau
$$

we understand an integral stretched on the set $0 \leqslant \tau_{i} \leqslant t_{i}(i=1, \ldots, m)$. The proof of Theorem $I$ is based on the Titchmarsh theorem, which holds for any number of dimensions (see [5]). This theorem permits to introduce the class of $m$-dimensional operators $a=p / q$, where $q$ does not vanish identically in the $m$-dimensional right neighbourhood of 0 . Then all the preceding considerations remain true in the new, more general, interpretation.

\section{References}

[1] C. Foiaş, Approximation des opérateurs de J. Milcusiński par des fonctions continues, Studia Math. 21 (1961), p. 73 - 74.

[2] J. Mikusiński, Operational Oalculus, 1959.

[3] - An approximation Theorem and its applications in Operational Oal. culus, Studia Math. 27 (1966), p. 141-145.

[4] - Germs and their Operational Oaloulus, ibidem 26 (1966), p. 315-325.

[5] - Oonvolution of functions of several variables, ibidem 20 (1961), p. $253-259$.

[6] K. Skórnik, A theorem on approximation in the olass $L^{p}$, Bull. Ac. Pol. Sci. 14,4 (1966), p. 195-196.

Regu par la Rédaction le 3. 1. 1964

\section{On generalized topological divisors of zero in $m$-convex locally convex algebras}

by

\section{W. ŻELAZKO (Warszawa)}

By a topological algebra we mean in this paper a topological linear space together with an associative jointly continuous multiplication. An element $x$ of a topological algebra $A, x \neq 0$, will be called a left (right) topological divisor of zero if there exists a non-void subset $P \subset A$ such that zero is not in the closure $\bar{P}$ of $P$ but $0 \epsilon \overline{x P}(0 \epsilon \overline{P x})$. Here, as usual, $U V$ $=\{x y: x \in U, y \in V\}$. An element $x \in A$ is called a topological divisor of zero in $A$ if it is both a right and a left topological divisor of zero. It is a classical fact of the theory of Banach algebras, due to Šilov [3] (for algebras without a unit, see [5]) that a complex Banach algebra either possesses topological divisors of zero or is isomorphically homeomorphic to the field of complex numbers. The same holds for locally bounded algebras a class more general than the class of Banach algebras [5]. Here we in vestigate the problem for another generalization of Banach algebras, namely for the class of locally convex multiplicatively convex topological algebras (shortly, we shall call them m-convex algebras throughout this paper). An m-convex algebra is a topological algebra (over complexes) with a basis for neighbourhoods of the origin consisting of sets $\{U\}$ which are convex, symmetric and idempotent, i. e. such that $U U \subset U$. Or, which is equivalent, it is a locally convex algebra with the topology given by means of family $\mathscr{P}$ of submultiplicative pseudonorms:

$$
\|x y\| \leqslant\|x\|\|y\|
$$

and, in the case where the algebra in question possesses a unit $e$,

$$
\|e\|=1 .
$$

for each $\|\cdot\| \epsilon \mathscr{P}$. We may assume that $\mathscr{P}$ consists of all continuous pseudonorms satisfying (1) and (2) in the case where there is a unit element. The theory of these algebras was created by Arens [1] and Michael [2].

The statement that an $m$-convex algebra either possesses topological divisors of zero or is isomorphically homeomorphic to the field of 